Molecules 2002, 7, 456-465

molecules

ISSN 1420-3049

http://www.mdpi.org

\title{
Niobium Pentachloride Activation of Enone Derivatives: Diels- Alder and Conjugate Addition Products
}

\section{Mauricio Gomes Constantino*, Valdemar Lacerda Júnior and Gil Valdo José da Silva}

Departamento de Química, Faculdade de Filosofia, Ciências e Letras de Ribeirão Preto, Universidade de São Paulo, Av. Bandeirantes 3900, 14040-901 - Ribeirão Preto - SP, Brazil. Phone +55/16 602 3747. Fax $+55 / 166338151$.

* Author to whom correspondence should be addressed. E-mail: mgconsta@usp.br; homepage: httpllartemis.ffclrp.usp.br

Received: 19 March 2002; in revised form: 15 May 2002 / Accepted: 16 May 2002 / Published: 31 May 2002

\begin{abstract}
Niobium pentachloride has proven to be a powerful activating agent for Diels-Alder or conjugate addition reactions of cycloenones. The Diels-Alder product was obtained only with an unsubstituted enone (cyclohexenone) and the highly reactive diene cyclopentadiene; substituents in the $\beta$-position of enones seem to prevent Diels-Alder reaction: oxygenated substituents favor the formation of vinyl chlorides (ethyl ether or dichloromethane as solvents) or enol ethers (ethyl acetate as solvent), while a methyl substituent prevents any kind of transformation with $\mathrm{NbCl}$. Less reactive dienes, furan and 2-methylfuran gave the conjugate addition products of the furan ring to the enone system.
\end{abstract}

Keywords: Niobium pentachloride, Lewis acid, Diels-Alder reaction, vinyl chlorides

\section{Introduction}

As part of our studies on the use of the Lewis acid niobium pentachloride in organic synthesis [1], we have recently started an investigation on Diels-Alder reactions catalysed with this reagent. As many Diels-Alder 
reactions proceed quite well without Lewis acids, the use of a catalyst can be justified only with less reactive substrates, or to obtain a different ratio of product isomers. In our investigations on the synthesis of natural products $[2,3]$ we found that a considerable simplification would be obtained if we could effect a Diels-Alder reaction between certain cyclic enones and dienes such as cyclopentadiene and furan derivatives. We have thus chosen cycloenones $\mathbf{1}, \mathbf{2}$ (the enolic form of 1,3-cyclohexanedione), 3, 4, 5 as dienophiles and compounds 6, 7 and $\mathbf{8}$ as dienes for our first studies. Under normal conditions these compounds do not react with each other to give Diels-Alder adducts; it is known, however, that some cycloenones do react with cyclopentadiene in the presence of $\mathrm{AlCl}_{3}$ [4]. The use of $\mathrm{NbCl}_{5}$ as Lewis acid for Diels-Alder reactions has also been previously reported [5,6].

Reactions were performed under nitrogen atmosphere, at room temperature or at $-78^{\circ} \mathrm{C}$, and in anhydrous solvents: ethyl acetate, dichloromethane or ethyl ether. Excess diene was used in each experiment, while the molar ratio dienophile/ $\mathrm{NbCl}$ was maintained constant in $1.0 / 0.5$.

Figure 1. Dienophiles and dienes used in experiments with $\mathrm{NbCl}_{5}$.

\section{Dienophiles}<smiles>O=C1C=CCCC1</smiles>

1<smiles>O=C1C=C(O)CCC1</smiles>

2<smiles>CC(=O)OC1=CC(=O)CCC1</smiles>

3

\section{Dienes}<smiles>C1=CCC=C1</smiles><smiles>c1ccoc1</smiles>

6 7<smiles>CC1=CC(=O)CC(C)(C)C1</smiles>

4<smiles>CC1=CC(=O)CCC1</smiles>

5

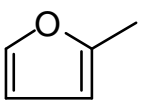

8

\section{Results and Discussion}

The reaction between 2-cyclohexenone (1) and cyclopentadiene (6) was effected under different conditions, as summarized in Scheme 1 and Table 1.

\section{Scheme 1}

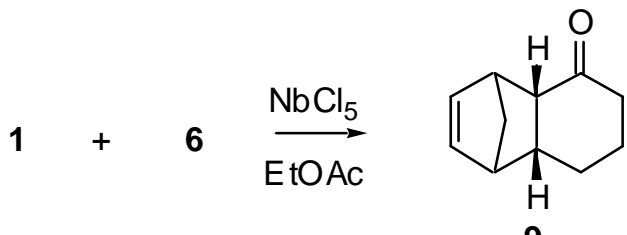


Table 1. Results obtained in the reaction of 2-cyclohexenone (1) and cyclopentadiene (6).

\begin{tabular}{cccc}
\hline $\mathbf{N b C l}_{\mathbf{5}}$ (eq.) & Temperature $\left({ }^{\circ} \mathbf{C}\right)$ & Time $(\mathbf{m i n})$ & Yield (\%) \\
\hline- & rt or reflux & 180 & - \\
0.5 & $\mathrm{rt}$ & 180 & 42 \\
0.5 & -78 & 90 & 74 \\
\hline
\end{tabular}

As shown by these results, no Diels-Alder reaction takes place in absence of catalyst, but addition of $\mathrm{NbCl}_{5}$ resulted in formation of Diels-Alder adduct either at room temperature or, in better yield, at $-78^{\circ} \mathrm{C}$. The yield improvement by lowering the temperature is a consequence of a decrease in diene polymerization The stereoselectivity of this reaction (only endo adduct $\mathbf{9}$ could be isolated) is a remarkable aspect, since when $\mathrm{AlCl}_{3}$ is used as catalyst [4] both endo and exo adducts are formed.

The other combinations of dienophile and diene from Figure 1 showed that, while a strong activation of the enone system by $\mathrm{NbCl}_{5}$ occurred in most cases, Diels-Alder adducts were not the products obtained.

Cycloenones 2-5 did not react with cyclopentadiene; only vinyl chlorides (10) or enol ethers (11) were obtained when these substrates were treated with cyclopentadiene and $\mathrm{NbCl}$. No product containing cyclopentadiene residues was isolated from these reaction mixtures. In fact, products $\mathbf{1 0}$ and $\mathbf{1 1}$ were obtained in better yields when the reaction was carried out in the absence of cyclopentadiene (Scheme 2 and Table 2). From these results it seems that substituents in the $\beta$ position of enones prevent the formation of Diels-Alder adducts: oxygenated substituents favor the formation of vinyl chlorides (when ethyl ether or dichloromethane are used as solvents) or enol ethers (when ethyl acetate is used as solvent), while methyl substituent prevents any reaction from taking place, starting material being recovered unchanged at the end of the reaction. A method for the preparation of vinyl chlorides and enol ethers can clearly be elaborated from these results, and we are already carrying out studies with this purpose.

Scheme 2
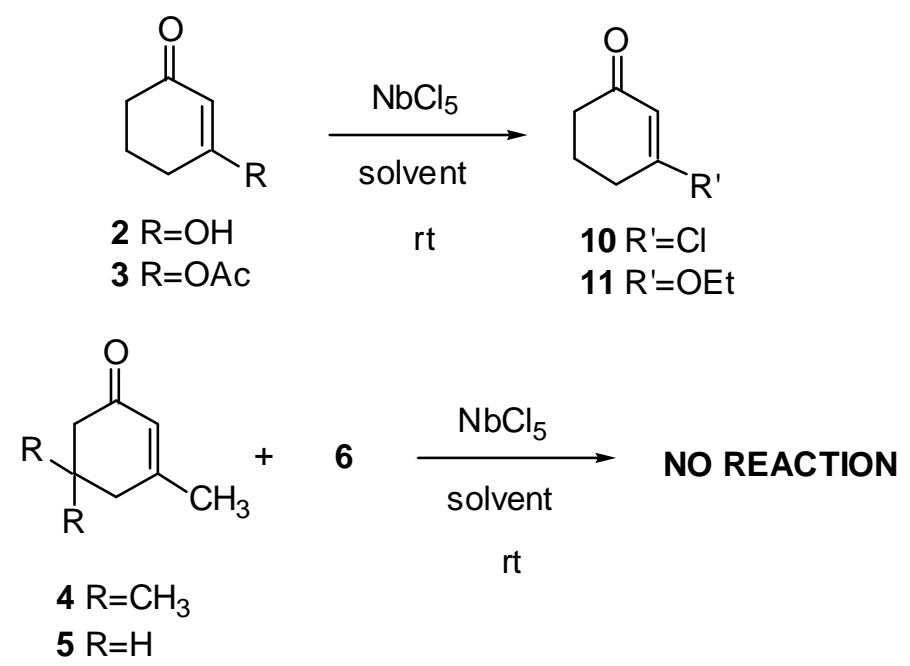
Table 2. Results of reaction of enones 2 - 5 with $\mathrm{NbCl}_{5}$ at room temperature.

\begin{tabular}{ccccc}
\hline \multirow{2}{*}{ Enone } & Solvent & Time (min.) & Yield (\%) & Ratio (\%) \\
\hline $\mathbf{2}$ & $\mathrm{Et}_{2} \mathrm{O}$ & 15 & 57 & $\mathbf{1 0} \mathbf{1 1}$ \\
$\mathbf{2}$ & $\mathrm{CH}_{2} \mathrm{Cl}_{2}$ & 30 & 68 & $100: 0$ \\
$\mathbf{2}$ & $\mathrm{EtOAc}$ & 30 & 60 & $100: 0$ \\
$\mathbf{3}$ & $\mathrm{Et}_{2} \mathrm{O}$ & 20 & 68 & $5: 95$ \\
$\mathbf{3}$ & $\mathrm{CH}_{2} \mathrm{Cl}_{2}$ & 20 & 80 & $100: 0$ \\
$\mathbf{3}$ & $\mathrm{EtOAc}$ & 30 & 83 & $100: 0$ \\
$\mathbf{4}^{\mathrm{a}}$ & $\mathrm{EtOAc}$ & & & $20: 80$ \\
$\mathbf{5}^{\mathrm{a}}$ & $\mathrm{EtOAc}$ & & & - \\
\hline
\end{tabular}

${ }^{a}$ These experiments were performed also under reflux and always in the presence of cyclopentadiene; no product was obtained even after several hours.

Reaction of furan (7) or 2-methylfuran (8) with enones $\mathbf{1}$ or $\mathbf{5}$ gave, rather than Diels-Alder adducts, products 12-16 (Scheme 3 and Table 3); under the same conditions, compound 4 did not react with furan 7.

\section{Scheme 3}<smiles>CCOC(C)(C)[N+](=O)[O-]</smiles>

12<smiles>CCOC(=O)CC1(c2ccc(C)o2)CCCC(c2ccc(C)o2)C1(C)c1ccc(C)o1</smiles>

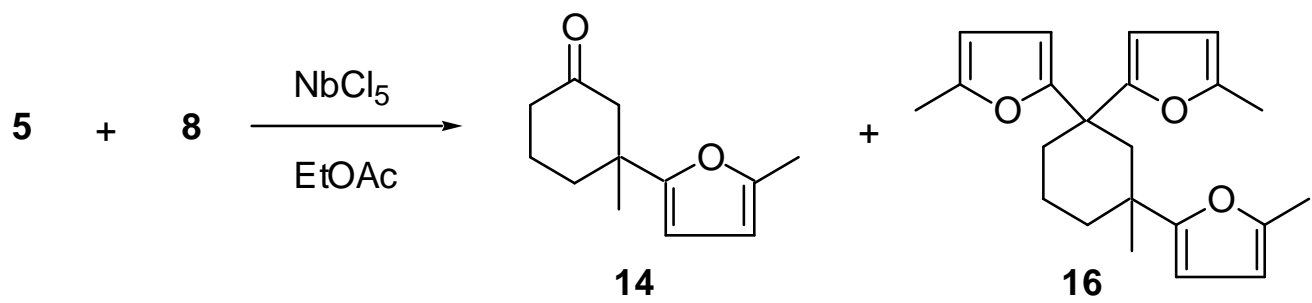


Table 3. Results obtained in reactions between enones 1,5 and furans 7,8 .

\begin{tabular}{cccccc}
\hline Dienophile & $\begin{array}{c}\text { Dien } \\
\text { e }\end{array}$ & Temperature ${ }^{\mathbf{O}} \mathbf{C}$ & Time (min) & Yield (\%) & Product (ratio,\%) \\
\hline $\mathbf{1}$ & $\mathbf{7}$ & $\mathrm{rt}$ & 15 & 66 & $\mathbf{1 2}$ only \\
$\mathbf{1}$ & $\mathbf{7}$ & -78 & 90 & 74 & $\mathbf{1 2}$ only \\
$\mathbf{1}$ & $\mathbf{8}$ & $\mathrm{rt}$ & 5 & 65 & $\mathbf{1 3}(33)$ and $\mathbf{1 5}(77)$ \\
$\mathbf{1}$ & $\mathbf{8}$ & -78 & 40 & 74 & $\mathbf{1 3}(95)$ and $\mathbf{1 5}(5)$ \\
$\mathbf{5}$ & $\mathbf{8}$ & $\mathrm{rt}$ & 300 & 68 & $\mathbf{1 4}(40)$ and $\mathbf{1 6}(60)$ \\
$\mathbf{5}$ & $\mathbf{8}$ & -78 & 600 & - & $\mathbf{1 4}^{\mathrm{a}}$ only \\
\hline
\end{tabular}

${ }^{\mathrm{a}}$ After 600 minutes only $50 \%$ of the starting material had been transformed.

Compounds 12-16 can be regarded either as products of conjugate (nucleophilic) addition of the furan ring to the enone system, or as products of electrophilic substitution of hydrogen by the enone system in furan ring, according to the mechanism for $\mathbf{1}$ and $\mathbf{8}$ shown in Scheme 4.

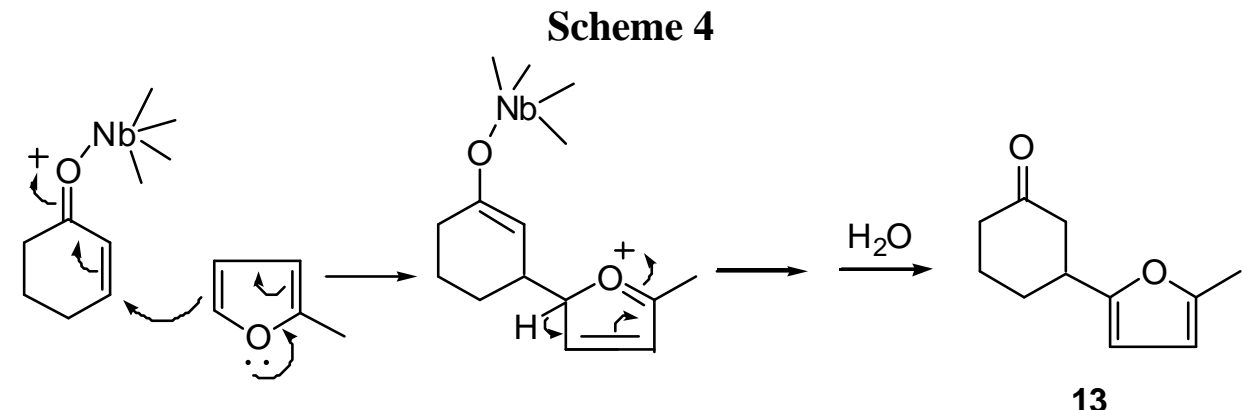

This type of reaction can be useful in synthesis of natural products; an obvious example is the preparation of an intermediate for the synthesis of nonactic acid by Beck and Henseleit [7], who used $\mathrm{BF}_{3}$ etherate as Lewis acid.

\section{Conclusions}

In one case, $\mathrm{NbCl}_{5}$ was shown to be an effective catalyst for Diels-Alder reaction, resulting in higher stereoselectivity as compared to $\mathrm{AlCl}_{3}$. When less reactive dienes and/or $\beta$-substituted cyclohexenones were used, however, no Diels-Alder adducts were obtained. Furan (7) and methylfuran (8) gave electrophilic substitution products with cyclohexenone (1) or 3-methylcyclohexenone (5); $\beta$-hydroxy-cyclohexenone (2) and $\beta$-acetoxycyclohexenone (3) reacted with $\mathrm{NbCl}_{5}$ to give a vinyl chloride or enol ether, depending on the solvent used and the addition of cyclopentadiene had no significant effect on these results; $\beta$ methylcyclohexenones $\mathbf{4}$ and $\mathbf{5}$ did not react, being recovered unchanged. Further studies can certainly establish more clear limits to the use of these reactions, each one being a useful transformation for organic synthesis. 


\section{Acknowledgments}

The authors thank the Fundação de Amparo à Pesquisa do Estado de São Paulo (FAPESP), the Conselho Nacional de Desenvolvimento Científico e Tecnológico (CNPq), and the Coordenadoria de Aperfeiçoamento de Pessoal do Ensino Superior (CAPES) for financial support and Dr. Adilson Beatriz for helpful comments and useful suggestions.

\section{Experimental}

\section{General}

NMR spectra were measured using a Bruker DPX-300 instrument (300 MHz for ${ }^{1} \mathrm{H}-\mathrm{NMR}$ and $75 \mathrm{MHz}$ for the ${ }^{13} \mathrm{C}-\mathrm{NMR}$ ) or a Bruker DRX-400 (400 MHz for ${ }^{1} \mathrm{H}-\mathrm{NMR}$ and $100 \mathrm{MHz}$ for the $\left.{ }^{13} \mathrm{C}-\mathrm{NMR}\right)$; deuterochloroform was used as solvent and tetramethylsilane as internal standard. GC-MS spectra were obtained by EI ionization at $70 \mathrm{eV}$ on a HP-5988-A spectrometer. IR spectra were measured with a PerkinElmer 1600 FT spectrometer. TLC was performed on precoated silica gel 60 F254 (0.25 mm thick, Merck), and for column chromatography silica gel 60, 70-230 mesh (Merck) was used. Analytical gas chromatography (GLC) separations were performed on a Varian GC 3400 instrument with a fused silica capillary column (30 m length x $0.25 \mathrm{~mm}$ i.d.) coated with DB 1701 (phase thickness $0.25 \mathrm{~mm}$ ) operating at temperatures in the range $50-200^{\circ} \mathrm{C}$.

\section{Preparation of substrates}

3-Acetoxy-2-cyclohexenone (3) [8]. A mixture of 1,3-cyclohexanedione (1.00 g, $8.93 \mathrm{mmol})$, acetic anhydride $(2 \mathrm{~mL})$, pyridine $(1.5 \mathrm{~mL})$ and 4dimethylaminopyridine $(10 \mathrm{mg})$ was stirred for 2 hat room temperature under nitrogen atmosphere. Ethyl ether $(20 \mathrm{~mL})$ was added and the organic layer was separated and washed with $10 \%$ aqueous hydrochloric acid $(3 \times 10 \mathrm{~mL})$, with saturated aqueous sodium bicarbonate $(2$ x $10 \mathrm{~mL})$, saturated brine $(2 \times 10 \mathrm{~mL})$, and dried over anhydrous magnesium sulfate. The solvent was removed under vacuum. Yield of compound 3 as yellow oil: $0.99 \mathrm{~g}(72 \%) ;{ }^{1} \mathrm{H}-\mathrm{NMR}(300 \mathrm{MHz}): \delta 5.89$ (t, $\left.1 \mathrm{H}, \mathrm{J}_{1}=\mathrm{J}_{2}=1.1 \mathrm{~Hz}\right), 2.54\left(\mathrm{td}, 2 \mathrm{H}, \mathrm{J}_{1}=6.2 \mathrm{~Hz}, \mathrm{~J}_{2}=1.1 \mathrm{~Hz}\right), 2.39\left(\mathrm{t}, 2 \mathrm{H}, \mathrm{J}_{1}=\mathrm{J}_{2}=6.2 \mathrm{~Hz}\right), 2.22(\mathrm{~s}, 3 \mathrm{H}), 2.05$ (qt, 2H, J = 6.2 Hz); ${ }^{13} \mathrm{C}-\mathrm{NMR}(75 \mathrm{MHz}): \delta 199.19(\mathrm{C}=\mathrm{O}), 169.52(\mathrm{C}), 166.92(\mathrm{C}=\mathrm{O}), 116.83(\mathrm{CH})$, $36.16\left(\mathrm{CH}_{2}\right), 27.78\left(\mathrm{CH}_{2}\right), 20.72\left(\mathrm{CH}_{3}\right), 20.72\left(\mathrm{CH}_{2}\right)$; IR (film) $v_{\max }: 2963,2887,1773,1675,1641$, 1429, 1361, 1196, $1124 \mathrm{~cm}^{-1}$; MS m/z (rel. intensity) (\%): 112 [(M-42) $]$ (4), 84 (23), 69 (9), 55 (4), 43 (100), 42 (11), 39 (11), 27 (10). 
3-Methyl-2-cyclohexenone (5) [9] To a solution of ethyl acetoacetate (12.6 g, $96.9 \mathrm{mmol})$ and formaldehyde $40 \%$ (3.67 g, $48.9 \mathrm{mmol}$ ) was added drop by drop a solution of piperidine $(0.3 \mathrm{~mL})$ in ethanol $(1.2 \mathrm{~mL})$, maintaining the temperature of the reaction below $30^{\circ} \mathrm{C}$ by cooling with a water bath containing some ice. Stirring was continued for $4 \mathrm{~h}$ and the mixture was then allowed to stand overnight. The upper water layer was separated and the lower oily layer was washed with water $(3 \times 15 \mathrm{~mL})$. The oily layer was mixed with $50 \mathrm{~mL}$ of $15 \%$ aqueous sulfuric acid and refluxed for $11 \mathrm{~h}$. The mixture was cooled and neutralized with ammonium hydroxide. The crude product was extracted with ethyl ether $(3 \times 15 \mathrm{~mL})$, dried over anhydrous magnesium sulfate and the solvent was removed under vacuum. The product was distilled (Kugelrohr) at $74{ }^{\circ} \mathrm{C} / 1.5$ mmHg to give $6.82 \mathrm{~g}(64 \%)$ of compound 5 as pale yellow oil: ${ }^{1} \mathrm{H}-\mathrm{NMR}(400 \mathrm{MHz}): \delta 5.86(\mathrm{~s}, 1 \mathrm{H}), 2.32$ (m, 4H), 2.01 (m, 2H), 1.97 (s, 3H).; ${ }^{13} \mathrm{C}-\mathrm{NMR}(100 \mathrm{MHz}): \delta 199.96(\mathrm{C}=\mathrm{O}), 163.21(\mathrm{C}), 126.87(\mathrm{CH})$, $37.89\left(\mathrm{CH}_{2}\right), 31.22\left(\mathrm{CH}_{2}\right), 24.74\left(\mathrm{CH}_{3}\right), 22.85\left(\mathrm{CH}_{2}\right)$; IR (film) $v_{\max }: 3039,2963,2872,1677,1629$, 1429, 1383, 1255, 1196, $1022 \mathrm{~cm}^{-1}$; MS m/z (rel. intensity) (\%): $110\left[\mathrm{M}^{+}\right]$(5), 82 (89), 67 (8), 54 (32), 41 (10), 39 (40), 32 (25), 28 (100).

General Procedure for the Reactions of Enones with $\mathrm{NbCl}_{5}$.

To a solution of $\mathrm{NbCl}_{5}(0.135 \mathrm{~g}, 0.5 \mathrm{mmol})$ in $1.0 \mathrm{~mL}$ of anhydrous solvent (ethyl acetate, ethyl ether or dichloromethane), maintained at room temperature or $-78^{\circ} \mathrm{C}$ under nitrogen atmosphere, was added a solution of the enone $(1.0 \mathrm{mmol})$ in $1.0 \mathrm{~mL}$ of the anhydrous solvent; in cases where a diene (cyclopentadiene or furans) was added, this solution contained also $2 \mathrm{mmol}$ of diene. The reaction mixture was quenched with a $10 \%$ aqueous citric acid solution $(2.0 \mathrm{~mL}$, when working at room temperature) or with a 1:1 solution of water/THF $\left(2.0 \mathrm{~mL}\right.$, when working at $\left.-78^{\circ} \mathrm{C}\right)$. The mixture was diluted with water $(5 \mathrm{~mL})$ and solvent $(10$ $\mathrm{mL})$, the organic layer was separated and washed with $5 \%$ aqueous sodium bicarbonate $(3 \times 10 \mathrm{~mL})$, saturated brine $(2 \times 10 \mathrm{~mL})$, and dried over anhydrous magnesium sulfate. The solvent was removed under vacuum and the products were purified by column chromatography through silica gel using a mixture of hexane and ethyl acetate (8:2) as eluent to give the products as yellow oils.

\section{Spectral Data}

Tricyclo[6.2.1.0 $0^{2,7}$ undec-9-en-3-one (9): ${ }^{1} \mathrm{H}-\mathrm{NMR}(300 \mathrm{MHz}): \delta 6.19\left(\mathrm{dd}, 1 \mathrm{H}, \mathrm{J}_{1}=5.7\right.$ and $\left.\mathrm{J}_{2}=2.9 \mathrm{~Hz}\right)$, $6.01\left(\mathrm{dd}, 1 \mathrm{H}, \mathrm{J}_{1}=5.7\right.$ and $\left.\mathrm{J}_{2}=2.9 \mathrm{~Hz}\right), 3.26$ (br. s, $\left.1 \mathrm{H}\right), 2.88$ (br. s, $\left.1 \mathrm{H}\right), 2.73\left(\mathrm{dd}, 1 \mathrm{H}, \mathrm{J}_{1}=10.2\right.$ and $\mathrm{J}_{2}=3.6$ $\mathrm{Hz}$ ), $2.67(\mathrm{~m}, 1 \mathrm{H}), 2.32$ (dddd, $1 \mathrm{H}, \mathrm{J}_{1}=18.4, \mathrm{~J}_{2}=6.0, \mathrm{~J}_{3}=2.5$ and $\mathrm{J}_{4}=1.9 \mathrm{~Hz}$ ), 2.03-1.92 (m, 1H), 1.93 (ddd, $1 \mathrm{H}, \mathrm{J}_{1}=18.4, \mathrm{~J}_{2}=12.0$ and $\left.\mathrm{J}_{3}=6.9 \mathrm{~Hz}\right), 1.85-1.62(\mathrm{~m}, 2 \mathrm{H}), 1.45\left(\mathrm{dt}, 1 \mathrm{H}, \mathrm{J}_{1}=8.3\right.$ and $\left.\mathrm{J}_{2}=\mathrm{J}_{3}=1.9 \mathrm{~Hz}\right), 1.31(\mathrm{dt}$, $1 \mathrm{H}, \mathrm{J}_{1}=8.3$ and $\left.\mathrm{J}_{2}=\mathrm{J}_{3}=1.5 \mathrm{~Hz}\right), 0.77$ (ddt, $1 \mathrm{H}, \mathrm{J}_{1}=\mathrm{J}_{2}=12.5, \mathrm{~J}_{3}=10.6$ and $\left.\mathrm{J}_{4}=3.5 \mathrm{~Hz}\right) ;{ }^{13} \mathrm{C}-\mathrm{NMR}(75 \mathrm{MHz}): \delta$ $215.47(\mathrm{C}=\mathrm{O}), 137.62(\mathrm{CH}), 134.91(\mathrm{CH}), 51.63(\mathrm{CH}), 48.32\left(\mathrm{CH}_{2}\right), 46.52(\mathrm{CH}), 45.19(\mathrm{CH}), 41.38$ $(\mathrm{CH}), 39.36\left(\mathrm{CH}_{2}\right), 27.97\left(\mathrm{CH}_{2}\right), 21.78\left(\mathrm{CH}_{2}\right)$; IR (film) $v_{\max }$ : 3060, 2932, 2857, 1696, 1607, 1455, 1337, 
1236, $1072 \mathrm{~cm}^{-1}$; MS m/z (rel. intensity) (\%):162 [M+'] (2), 121 (2), 97 (39), 91 (19), 79 (15), 66 (100), 43 (8), 41 (16).

3-Chloro-2-cyclohexenone (10): ${ }^{1} \mathrm{H}-\mathrm{NMR}(300 \mathrm{MHz}): \delta 6.22(\mathrm{t}, 1 \mathrm{H}, \mathrm{J}=1.5 \mathrm{~Hz}), 2.69\left(\mathrm{td}, 2 \mathrm{H}, \mathrm{J}_{1}=\mathrm{J}_{2}=6.5\right.$ and $\left.\mathrm{J}_{3}=1.5 \mathrm{~Hz}\right), 2.40(\mathrm{t}, 2 \mathrm{H}, \mathrm{J}=6.5 \mathrm{~Hz}), 2.09(\mathrm{q}, 2 \mathrm{H}, \mathrm{J}=6.5 \mathrm{~Hz}) ;{ }^{13} \mathrm{C}-\mathrm{NMR}(75 \mathrm{MHz}): \delta 196.85(\mathrm{C}=\mathrm{O})$, $158.56(\mathrm{C}), 128.44(\mathrm{CH}), 36.29\left(\mathrm{CH}_{2}\right), 33.86\left(\mathrm{CH}_{2}\right), 22.17\left(\mathrm{CH}_{2}\right)$; IR (film) v $v_{\max }: 3041,2948,2887$, $1679,1607,1455,1425,1344,1289,1234,1137,1026 \mathrm{~cm}^{-1}$; MS m/z (rel. intensity) (\%):132 [(M+2) $]$ (10), 130 (34), 104 (32), 102 (100), 67 (75), 65 (9), 39 (43), 28 (11).

3-Ethoxy-2-cyclohexenone (11): ${ }^{1} \mathrm{H}-\mathrm{NMR}(300 \mathrm{MHz}): \delta 5.34(\mathrm{~s}, 1 \mathrm{H}), 3.91(\mathrm{q}, 2 \mathrm{H}, \mathrm{J}=7.0 \mathrm{~Hz}), 2.41(\mathrm{t}$, $2 \mathrm{H}, \mathrm{J}=6.4 \mathrm{~Hz}), 2.33(\mathrm{t}, 2 \mathrm{H}, \mathrm{J}=6.4 \mathrm{~Hz}), 1.98(\mathrm{q}, 2 \mathrm{H}, \mathrm{J}=6.4 \mathrm{~Hz}), 1.37$ (t, 3H, J=7.0 Hz), ${ }^{13} \mathrm{C}-\mathrm{NMR}(75$ MHz): $\delta 199.69(\mathrm{C}=\mathrm{O}), 177.92(\mathrm{C}), 102.67(\mathrm{CH}), 65.18\left(\mathrm{CH}_{2}\right), 36.78\left(\mathrm{CH}_{2}\right), 29.11\left(\mathrm{CH}_{2}\right), 22.29\left(\mathrm{CH}_{2}\right)$, $14.14\left(\mathrm{CH}_{3}\right)$; IR (film) $v_{\max }: 3039,2948,2887,1646,1599,1465,1221,1137,1026 \mathrm{~cm}^{-1}$; MS m/z (rel. intensity) (\%): 112 [(M-28) $\left.{ }^{+}\right](28), 84$ (81), 69 (23), 55 (35), 42 (100), 39 (37), 27 (37), 15 (9).

3-[5-(3-Oxo-cyclohexyl)-furan-2-yl]-cyclohexanone (12): ${ }^{1} \mathrm{H}-\mathrm{NMR}(300 \mathrm{MHz}): \delta 5.91$ (s, 2H), 3.15 (ddt, $2 \mathrm{H}, \mathrm{J}_{1}=10.5, \mathrm{~J}_{2}=8.6$ and $\mathrm{J}_{3}=\mathrm{J}_{4}=4.8 \mathrm{~Hz}$ ), $2.66\left(\mathrm{ddt}, 2 \mathrm{H}, \mathrm{J}_{1}=14.2, \mathrm{~J}_{2}=4.8\right.$ and $\mathrm{J}_{3}=\mathrm{J}_{4}=0.9 \mathrm{~Hz}$ ), 2.49 (ddd, $2 \mathrm{H} ; \mathrm{J}_{1}=14.2, \mathrm{~J}_{2}=10.5$ and $\left.\mathrm{J}_{3}=0.9 \mathrm{~Hz}\right), 2,35(\mathrm{~m}, 4 \mathrm{H}), 2.10(\mathrm{~m}, 4 \mathrm{H}), 1.82(\mathrm{~m}, 4 \mathrm{H}) ;{ }^{13} \mathrm{C}-\mathrm{NMR}(75$ MHz): $\delta 210.11(\mathrm{C}=\mathrm{O}), 155.81(\mathrm{C}), 104.80(\mathrm{CH}), 45.51\left(\mathrm{CH}_{2}\right), 41.16\left(\mathrm{CH}_{2}\right), 37.49(\mathrm{CH}), 29.77\left(\mathrm{CH}_{2}\right)$, $24.26\left(\mathrm{CH}_{3}\right)$; IR (film) $v_{\max }: 3041,3037,2948,2872,1709,1599,1557,1455,1421,1221,1175,1099$, $1018 \mathrm{~cm}^{-1}$; MS m/z (rel. intensity) (\%): $260\left[\mathrm{M}^{+}\right]$(2), 203 (3), 55 (3), 42 (4), 41 (3), 32 (20), 28 (100), 27 (2).

(士)-3-(5-Methyl-furan-2-yl)-cyclohexanone (13): ${ }^{1} \mathrm{H}-\mathrm{NMR}(400 \mathrm{MHz}): \delta 5.88(\mathrm{~d}, 1 \mathrm{H}, \mathrm{J}=3.3 \mathrm{~Hz}), 5.85(\mathrm{~d}$, $1 \mathrm{H}, \mathrm{J}=3.3 \mathrm{~Hz}$ ), $3.12\left(\mathrm{tt}, 1 \mathrm{H}, \mathrm{J}_{1}=\mathrm{J}_{2}=10.0\right.$ and $\left.\mathrm{J}_{3}=\mathrm{J}_{4}=4.5 \mathrm{~Hz},\right), 2.66\left(\mathrm{ddt}, 1 \mathrm{H}, \mathrm{J}_{1}=14.5, \mathrm{~J}_{2}=4.5\right.$ and $\mathrm{J}_{3}=\mathrm{J}_{4}=1.6 \mathrm{~Hz}$ ), $2.51\left(\mathrm{ddd}, 1 \mathrm{H}, \mathrm{J}_{1}=14.5, \mathrm{~J}_{2}=10,0\right.$ and $\left.\mathrm{J}_{3}=1.3 \mathrm{~Hz}\right), 2.37(\mathrm{~m}, 2 \mathrm{H}), 2.25(\mathrm{~s}, 3 \mathrm{H}), 2.15(\mathrm{~m}, 1 \mathrm{H})$, $2.01(\mathrm{~m}, 1 \mathrm{H}), 1.82(\mathrm{~m}, 2 \mathrm{H}) ;{ }^{13} \mathrm{C}-\mathrm{NMR}(100 \mathrm{MHz}): \delta 210.41(\mathrm{C}=\mathrm{O}), 155.38(\mathrm{C}), 150.39(\mathrm{C}), 105.80$ $(\mathrm{CH}), 105.05(\mathrm{CH}), 45.75\left(\mathrm{CH}_{2}\right), 41.27\left(\mathrm{CH}_{2}\right), 37.65(\mathrm{CH}), 30.04\left(\mathrm{CH}_{2}\right), 24.41\left(\mathrm{CH}_{2}\right), 13.47\left(\mathrm{CH}_{3}\right)$; IR (film) $v_{\max }: 3115,2948,2872,1713,1616,1569,1446,1319,1221,1022 \mathrm{~cm}^{-1}$; MS m/z (rel. intensity) (\%): $178\left[\mathrm{M}^{+}\right](6), 121$ (15), 108 (10), 77 (4), 55 (6), 42 (13), 32 (28), 28 (100).

(士)-3-Methyl-3-(5-methyl-furan-2-yl)-cyclohexanone (14): ${ }^{1} \mathrm{H}-\mathrm{NMR}(400 \mathrm{MHz}): \delta 5.86(\mathrm{~d}, 1 \mathrm{H}, \mathrm{J}=3.0$ $\mathrm{Hz}), 5.81(\mathrm{~d}, 1 \mathrm{H}, \mathrm{J}=3.0 \mathrm{~Hz}), 2.71\left(\mathrm{dt}, 1 \mathrm{H}, \mathrm{J}_{1}=14.0\right.$ and $\left.\mathrm{J}_{2}=\mathrm{J}_{3}=1.5 \mathrm{~Hz}\right), 2.31(\mathrm{~m}, 3 \mathrm{H}), 2.23(\mathrm{~s}, 3 \mathrm{H}), 2.20(\mathrm{~m}$, $1 \mathrm{H}), 1.86(\mathrm{~m}, 1 \mathrm{H}), 1.73(\mathrm{~m}, 1 \mathrm{H}), 1.64(\mathrm{~m}, 1 \mathrm{H}), 1.30(\mathrm{~m}, 3 \mathrm{H}) ;{ }^{13} \mathrm{C}-\mathrm{NMR}(100 \mathrm{MHz}): \delta 210.79(\mathrm{C}=\mathrm{O})$, $157.89(\mathrm{C}), 150.79(\mathrm{C}), 105.68(\mathrm{CH}), 105.64(\mathrm{CH}), 51.88\left(\mathrm{CH}_{2}\right), 40.71\left(\mathrm{CH}_{2}\right), 40.44(\mathrm{C}), 35.91\left(\mathrm{CH}_{2}\right)$, $27.18\left(\mathrm{CH}_{3}\right), 22.06\left(\mathrm{CH}_{2}\right), 13.47\left(\mathrm{CH}_{3}\right)$; IR (film) $v_{\max }:$ 3115, 2948, 2857, 1709, 1607, 1557, 1455, 1349, 1221, 1115, $1022 \mathrm{~cm}^{-1}$; MS m/z (rel. intensity) (\%): $192\left[\mathrm{M}^{+}\right]$(4), 149 (4), 135 (10), 122 (8), 98 (3), 77 (4), 55 (35), 28 (100). 
(士)-2-[3,3-Bis-(5-methyl-(2-furan-2-yl)-cyclohexyl]-5-methyl-furan (15): ${ }^{1} \mathrm{H}-\mathrm{NMR}(400 \mathrm{MHz}): \delta 6.11$ $(\mathrm{d}, 1 \mathrm{H} ; \mathrm{J}=3.0 \mathrm{~Hz}), 5.90(\mathrm{~d}, 1 \mathrm{H}, \mathrm{J}=3.0 \mathrm{~Hz}), 5.84(\mathrm{~d}, 1 \mathrm{H}, \mathrm{J}=3.3 \mathrm{~Hz}), 5.81(\mathrm{~d}, 1 \mathrm{H}, \mathrm{J}=3.3 \mathrm{~Hz}), 5.76(\mathrm{~d}, 1 \mathrm{H}$, $\mathrm{J}=3.0 \mathrm{~Hz}), 5.64(\mathrm{~d}, 1 \mathrm{H}, \mathrm{J}=3.0 \mathrm{~Hz}), 2.81\left(\mathrm{tt}, 1 \mathrm{H}, \mathrm{J}_{1}=\mathrm{J}_{2}=12.0\right.$ and $\left.\mathrm{J}_{3}=\mathrm{J}_{4}=3.3 \mathrm{~Hz}\right), 2.74(\mathrm{~m}, 1 \mathrm{H}), 2.48(\mathrm{~m}, 1 \mathrm{H})$, $2.23(\mathrm{~s}, 3 \mathrm{H}), 2.21(\mathrm{~s}, 3 \mathrm{H}), 2.80(\mathrm{~s}, 3 \mathrm{H}), 1.95(\mathrm{~m}, 1 \mathrm{H}), 1.92(\mathrm{t}, 1 \mathrm{H}, \mathrm{J}=12.0 \mathrm{~Hz}), 1.84\left(\mathrm{dd}, 1 \mathrm{H}, \mathrm{J}_{1}=12.0\right.$ and $\left.\mathrm{J}_{2}=3.5 \mathrm{~Hz}\right), 1.73(\mathrm{~m}, 1 \mathrm{H}), 1.55\left(\mathrm{ddt}, 1 \mathrm{H}, \mathrm{J}_{1}=25.0, \mathrm{~J}_{2}=14.0\right.$ and $\left.\mathrm{J}_{3}=\mathrm{J}_{4}=3.5 \mathrm{~Hz}\right), 1.37\left(\mathrm{dd}, 1 \mathrm{H}, \mathrm{J}_{1}=25.0\right.$, $\left.\mathrm{J}_{2}=12.5 \mathrm{~Hz}\right),{ }^{13} \mathrm{C}-\mathrm{NMR}(100 \mathrm{MHz}): \delta 159.88(\mathrm{C}), 159.02(\mathrm{C}), 155.06(\mathrm{C}), 151.07$ (C), $150.70(\mathrm{C})$, $150.43(\mathrm{C}), 108.06(\mathrm{CH}), 106.60(\mathrm{CH}), 106.23(\mathrm{CH}), 106.14(\mathrm{CH}), 104.66(\mathrm{CH}), 103.96(\mathrm{CH}), 42.40$ (C), $39.12\left(\mathrm{CH}_{2}\right), 33.85\left(\mathrm{CH}_{2}\right), 33.81(\mathrm{CH}), 31.69\left(\mathrm{CH}_{2}\right), 22.70\left(\mathrm{CH}_{2}\right), 14.10\left(\mathrm{CH}_{3}\right), 14.04\left(\mathrm{CH}_{3}\right), 13.97$ $\left(\mathrm{CH}_{3}\right)$; IR (film) $v_{\text {max }}: 3115,2948,2872,1612,1557,1450,1378,1217,1026 \mathrm{~cm}^{-1}$; MS m/z (rel. intensity) (\%): $242\left[(\mathrm{M}-82)^{+}\right](8), 199$ (7), 188 (4), 108 (5), 95 (5), 61 (4), 43 (53), 28 (100).

(士)-2-[1,3-Bis(5-methyl-(2-furan-2-yl))-3-methyl-cyclohexyl]-5-methyl-furan (16): ${ }^{1} \mathrm{H}-\mathrm{NMR}(400 \mathrm{MHz})$ : $\delta 5.91(\mathrm{~d}, 1 \mathrm{H} ; \mathrm{J}=3.0 \mathrm{~Hz}), 5.87$ (d, 1H, J=3.0 Hz), 5.75 (s, 2H), 5.72 (d, 1H, J=3.0 Hz), 5.62 (d, 1H, J=3.0 Hz), 2.47 (d, 1H, J=13.5 Hz), 2.42 (d, 1H, J=13.5), 2.25 (s, 3H), 2.23 (s, 3H), $2.23(\mathrm{~m}, 1 \mathrm{H}), 2.17$ (s, 3H), $2.02(\mathrm{~m}, 1 \mathrm{H}), 1.83(\mathrm{~m}, 3 \mathrm{H}), 1.59(\mathrm{~m}, 1 \mathrm{H}), 0.96(\mathrm{~s}, 3 \mathrm{H}) ;{ }^{13} \mathrm{C}-\mathrm{NMR}(100 \mathrm{MHz}): \delta 162.25(\mathrm{C}), 158.26(\mathrm{C})$, $156.66(\mathrm{C}), 150.09(\mathrm{C}), 150.00(\mathrm{C}), 149.53(\mathrm{C}), 106.08(\mathrm{CH}), 105.63(\mathrm{CH}), 105.51(\mathrm{CH}), 105.35(\mathrm{CH})$, $104.78(\mathrm{CH}), 102.64(\mathrm{CH}), 42.50\left(\mathrm{CH}_{2}\right), 40.54(\mathrm{C}), 36.00\left(\mathrm{CH}_{2}\right), 35.63(\mathrm{C}), 33.45\left(\mathrm{CH}_{2}\right), 25.32\left(\mathrm{CH}_{3}\right)$, $19.07\left(\mathrm{CH}_{2}\right), 13.63\left(\mathrm{CH}_{3}\right), 13.57\left(\mathrm{CH}_{3}\right), 13.55\left(\mathrm{CH}_{3}\right)$; IR (film) $v_{\max }: 3100,2948,2842,1612,1561$, 1450, 1319, 1221, $1022 \mathrm{~cm}^{-1}$; MS m/z (rel. intensity) (\%): 338 [(M) $\left.{ }^{+}\right](2), 188(5), 175$ (5), 135 (6), 122 (6), 95 (4), 91 (4), 28 (100).

\section{References and Notes}

1. Constantino, M. G.; Lacerda Jr., V.; Aragão, V. Molecules 2001, 6, 770-776.

2. Constantino, M. G.; Beatriz, A.; da Silva, G. V. J. Tetrahedron Lett. 2000, 41, 7001-7004

3. Constantino, M. G.; Beatriz, A.; da Silva, G. V. J.; Zukerman-Schpector, J. Synth. Comm. 2001, 31, 3329-3336.

4. Angell, E. C.; Fringuelli, F.; Guo, M.; Minuti, L.; Taticchi, A.; Wenkert, E. J. Org. Chem. 1988, 53, 4325-4328.

5. Howarth, J.; Gillespie, K. Tetrahedron Lett. 1996, 37, 6011-6012.

6. Howarth, J.; Gillespie, K. Molecules 2000, 5, 993-997.

7. Beck, G.; Henseleit, E. Chem. Ber. 1971, 104, 21-29.

8. Hofle, G.; Steglich, W.; Vorbrugzen, H. Angew. Chem. 1978, 17, 569-574.

9. Natelson, S.; Gottfried, S. P. J. Am. Chem. Soc. 1939, 61, 1001-1002.

Sample Availability: Samples of compounds 1-16 are available from MDPI.

(C) 2002 by MDPI (http://www.mdpi.org). Reproduction is permitted for non commercial purposes. 